Honam Mathematical J. 34 (2012), No. 4, pp. 513-517

http://dx.doi.org/10.5831/HMJ.2012.34.4.513

\title{
HORIZONTAL SUBSPACES IN THE BUNDLE OF LINEAR FRAMES
}

\author{
JOON-SIK PARK
}

\begin{abstract}
Let $L(M)$ be the bundle of all linear frames over a smooth manifold $M, u$ an arbitrarily given point of $L(M)$, and $\nabla: \mathfrak{X}(M) \times \mathfrak{X}(M) \rightarrow \mathfrak{X}(M)$ a linear connection on $M$. Then the following result is well known: the horizontal subspace at the point $u$ may be written in terms of local coordinates of $u \in L(M)$ and Christoffel's symbols defined by $\nabla$. This result is very fundamental on the study of the theory of connections. In this paper we show that the local expression of the horizontal subspace at the point $u$ does not depend on the choice of a local coordinate system around the point $u \in L(M)$, which is rarely seen.
\end{abstract}

\section{$\S 1$. Introduction}

Let $L(M)$ be the bundle of all linear frames over a smooth manifold $M, u$ an arbitrarily given point of $L(M)$, and $\nabla: \mathfrak{X}(M) \times \mathfrak{X}(M) \rightarrow \mathfrak{X}(M)$ a linear connection on $M$. Here $\mathfrak{X}(M)$ is the set of all smooth vector fields on $M$. In this paper, in terms of local coefficient functions $\Gamma_{j k}^{i}$ of the linear connection $\nabla$ on $M$ and local coordinates of the point $u \in L(M)$, we express the horizontal subspace $Q_{u} \subset T_{u}(L(M))$. This result is well known, and is very fundamental in the study on the theory of connections. In this paper we show that the local expression of the horizontal subspace at the point $u$ does not depend on the choice of a local coordinate system around the point $u \in L(M)$, which is rarely seen. Moreover, we give full explanations for the above consequence.

\section{$\S 2$. Horizontal subspaces in the bundle of linear frames over a smooth manifold}

Received August 15, 2012. Accepted September 7, 2012.

2010 Mathematics Subject Classification. 53C07; 53A15.

Key words and phrases. bundle of linear frames, connection, horizontal subspace..

This work was supported by the research grant of the Busan University of Foreign Studies in 2012 . 
2.1. Connections in a principal fiber bundle. Let $(P, M, G, \pi)$ be a principal fiber bundle over a manifold $M$ with group $G$. For each $u \in P$, let $T_{u}(P)$ be the tangent space of $P$ at $u$ and $G_{u}$ the subspace of $T_{u}(P)$ consisting of vectors tangent to the fiber through $u$. A connection in $(P, M, G, \pi)$ is an assignment of a subspace $Q_{u}$ of $T_{u}(P)$ to each $u \in P$ such that

$$
T_{u}(P)=G_{u}+Q_{u}(\text { direct sum }) ;
$$

(2.2) $Q_{u g}=R_{g_{\star}} Q_{u}$ for every $u \in P$ and $g \in G$, where $R_{g}$ is the transformation of $P$ induced by $g \in G, R_{g} u=u g$;

(2.3) $Q_{u}$ depends differentiably on $u$.

Given a connection $Q$ in $(P, M, G, \pi)$, we define a 1 -form $\omega$ on $P$ with values in the Lie algebra $\mathfrak{g}$ of $G$ as follows. For each $W \in T_{u} P$, we define $\omega(W)$ to be the unique $x \in \mathfrak{g}$ such that $\left(x^{\star}\right)_{u}$ is equal to the vertical component of $W$. Here $x^{\star}$ is the fundamental vector field corresponding to $x \in \mathfrak{g}$ which is defined on $P$ (cf. $[1,2])$. The form $\omega$ is called the connection form of the given connection $Q$ (cf. $[2,3,4]$ ).

2.2. The Christoffel's symbols of a linear connection on $M$. Let $\nabla: \mathfrak{X}(M) \times \mathfrak{X}(M) \rightarrow \mathfrak{X}(M)$ be a linear connection on $M$. Taking local coordinate systems $\left(x^{1}, \ldots, x^{n}\right),\left(y^{1}, \ldots, y^{n}\right)$ on neighborhoods $U, V$ being contained in $M$ respectively, then we may write

$$
\begin{aligned}
& \nabla_{\frac{\partial}{\partial x^{j}}} \frac{\partial}{\partial x^{i}}=\sum_{k=1}^{n} \Gamma_{j i}^{k} \frac{\partial}{\partial x^{k}} \\
& \nabla_{\frac{\partial}{\partial y^{j}}} \frac{\partial}{\partial y^{i}}=\sum_{k=1}^{n} \tilde{\Gamma}_{j i}^{k} \frac{\partial}{\partial y^{k}}
\end{aligned}
$$

on $U, V$ respectively. Here $\Gamma_{j i}^{k}$ (resp. $\tilde{\Gamma}_{j i}^{k}$ ) are called the Christoffel's symbols for the linear connection $\nabla$ on $M$ relative to the local coordinate system $\left(x^{1}, \ldots, x^{n}\right)$ (resp. $\left.\left(y^{1}, \ldots, y^{n}\right)\right)$ on neighborhoods $U$ (resp. $V$ ) on $M$. In the intersection of the two coordinate neighborhoods $U$ and $V$, we have

$$
\tilde{\Gamma}_{j i}^{k}=\sum_{l, s, h} \frac{\partial y^{k}}{\partial x^{l}} \frac{\partial x^{s}}{\partial y^{j}} \frac{\partial x^{h}}{\partial y^{i}} \Gamma_{s h}^{l}+\sum_{l} \frac{\partial^{2} x^{l}}{\partial y^{j} \partial y^{i}} \frac{\partial y^{k}}{\partial x^{l}} .
$$

2.3. Horizontal subspaces and connection form. Here and from now on in this paper, we shall denote the bundle $L(M)$ of linear frames on $M$ by $P$ and the general linear group $G L(n, \mathbb{R}), n=\operatorname{dim} M$, by $G$. Let $\nabla: \mathfrak{X}(M) \times \mathfrak{X}(M) \rightarrow \mathfrak{X}(M)$ be a linear connection on $M$. Let $\Gamma_{j i}^{k}$ and $\tilde{\Gamma}_{j i}^{k}$ the Christoffel's symbols with respect to local coordinate 
systems $x^{1}, \ldots, x^{n}, y^{1}, \ldots, y^{n}$ which are defined on neighborhoods $U, V$, respectively. For $w \in \pi^{-1}(U \cap V)$ we may write

$$
\begin{aligned}
w & =\left(\frac{\partial}{\partial x^{1}}, \ldots, \frac{\partial}{\partial x^{n}}\right)_{\pi(w)}\left(a_{j}^{i}(\pi(w))\right)_{i, j} \\
& =\left(\frac{\partial}{\partial y^{1}}, \ldots, \frac{\partial}{\partial y^{n}}\right)_{\pi(w)}\left(b_{j}^{i}(\pi(w))\right)_{i, j} .
\end{aligned}
$$

So we obtain two local coordinate systems on $\pi^{-1}(U \cap V)$

$$
\left(x^{1}, \ldots, x^{n}, a_{1}^{1}, \ldots, a_{n}^{n}\right) \text { and }\left(y^{1}, \ldots, y^{n}, b^{1}{ }_{1}, \ldots, b_{n}^{n}\right) .
$$

Let $p$ be an arbitrarily given point which belongs to $U$, and let $X=$ $\sum_{i} \xi^{i}\left(\partial / \partial x^{i}\right)_{p}=\sum_{i} \eta^{i}\left(\partial / \partial y^{i}\right)_{p},\left(\xi^{i}, \eta^{i} \in \mathbb{R}\right)$, be an arbitrarily given tangent vector which belongs to $T_{p}(M)$. Then

(2.8) $\eta^{i}=\sum_{j} \xi^{j}\left(\partial y^{i} / \partial x^{j}\right)_{p} \in \mathbb{R}$.

First of all, we obtain the horizontal lift of $X$ at $u \in P,(\pi(u)=p)$. Let $c_{t},-\epsilon<t<\epsilon$, be an integral curve of $X$ which satisfies the following conditions:

$$
\begin{aligned}
& c_{0}=p=\pi(u), \\
& \left(x^{1}\left(c_{t}\right), \ldots, x^{n}\left(c_{t}\right)\right)=\left(\xi^{1} t, \ldots, \xi^{n} t\right) \quad(-\epsilon<t<\epsilon) .
\end{aligned}
$$

Let $u_{t},-\epsilon<t<\epsilon$, be the horizontal lift of $c_{t}$ satisfying the initial condition $u_{0}=u$. We denote $u_{t},-\epsilon<t<\epsilon$, by $\left(X_{1}(t), \ldots, X_{n}(t)\right)$. Then, $\left(X_{1}(0), \ldots, X_{n}(0)\right)=u_{0}=u$. Moreover we may write for each $t \in(-\epsilon, \epsilon)$

$$
\begin{aligned}
u_{t} & =\left(X_{1}(t), \ldots, X_{n}(t)\right) \\
& =\left(\frac{\partial}{\partial x^{1}}, \ldots, \frac{\partial}{\partial x^{n}}\right)_{c_{t}}\left(a^{i}{ }_{j}\left(u_{t}\right)\right)_{i, j} \\
& =\left(\frac{\partial}{\partial y^{1}}, \ldots, \frac{\partial}{\partial y^{n}}\right)_{c_{t}}\left(b^{i}{ }_{j}\left(u_{t}\right)\right)_{i, j} .
\end{aligned}
$$

From (2.6) we get on $\pi^{-1}(U \cap V)$

$$
b_{j}^{i}=\sum_{l} \frac{\partial y^{i}}{\partial x^{l}} a_{j}^{l}, a_{j}^{i}=\sum_{l} \frac{\partial x^{i}}{\partial y^{l}} b_{j}^{l} .
$$

In order to obtain the main result, we recall the lemma below

Lemma 2.1 [2]. Let $v_{t}=\left(Y_{1}(t), \ldots, Y_{n}(t)\right),-\epsilon<t<\epsilon$, be a smooth curve in $L(M)$ with $\pi\left(v_{t}\right)=c(t)$. Then the following statements are equivalent:

(i) each $Y_{i}(t),-\epsilon<t<\epsilon$, is parallel along the curve $c(t)$;

(ii) $v_{t},-\epsilon<t<\epsilon$, is the horizontal lift of the curve $c(t)$ passing through the point $v_{0}$. 
Since $u_{t},-\epsilon<t<\epsilon$, is the horizontal curve of the curve $c_{t}$ with $u_{0}=u$, we get from (2.4), (2.9) and Lemma 2.1

$$
\begin{aligned}
\nabla_{d c_{t} / d t} X_{j}(t) & =\sum_{i} \nabla_{d c_{t} / d t} a_{j}^{i}\left(u_{t}\right)\left(\partial / \partial x^{i}\right)_{c_{t}} \\
& =\sum_{i}\left\{d a_{j}^{i}\left(u_{t}\right) / d t+\sum_{k, l} \xi^{l} a^{k}{ }_{j}\left(u_{t}\right) \Gamma_{l k}^{i}\right\}\left(\partial / \partial x^{i}\right)_{c_{t}}=0 .
\end{aligned}
$$

So the horizontal lift $X^{\star} \in T_{u}(P)$ of the vector $X=\sum_{i} \xi^{i}\left(\partial / \partial x^{i}\right)_{p}$ may be written as

$$
X^{\star}=\sum_{i} \xi^{i}\left(\partial / \partial x^{i}\right)_{u}-\sum_{i, l} \xi^{i} a_{j}^{l}(u) \Gamma_{i l}^{k}(p)\left(\partial / \partial a_{j}^{k}\right)_{u} .
$$

By virtue of (2.6) and (2.7), we get on $\pi^{-1}(U \cap V)$

$$
\begin{aligned}
& \frac{\partial}{\partial x^{k}}=\sum_{i} \frac{\partial y^{i}}{\partial x^{k}} \frac{\partial}{\partial y^{i}}+\sum_{h, i, l} a^{h}{ }_{l} \frac{\partial^{2} y^{i}}{\partial x^{k} \partial x^{h}} \frac{\partial}{\partial b^{i}{ }_{l}}, \quad \frac{\partial}{\partial a^{s}{ }_{j}}=\sum_{i} \frac{\partial y^{i}}{\partial x^{s}} \frac{\partial}{\partial b^{i}{ }_{j}}, \\
& d x^{i}=\sum_{j} \frac{\partial x^{i}}{\partial y^{j}} d y^{j}, \quad d a^{i}{ }_{j}=\sum_{k, l} \frac{\partial^{2} x^{i}}{\partial y^{k} \partial y^{l}} b_{j}^{l} d y^{k}+\sum_{l} \frac{\partial x^{i}}{\partial y^{l}} d b_{j}^{l}, \\
& \frac{\partial}{\partial y^{k}}=\sum_{i} \frac{\partial x^{i}}{\partial y^{k}} \frac{\partial}{\partial x^{i}}+\sum_{h, i, l} b^{h}{ }_{l} \frac{\partial^{2} x^{i}}{\partial y^{k} \partial y^{h}} \frac{\partial}{\partial a^{i} l_{l}}, \quad \frac{\partial}{\partial b^{s}{ }_{j}}=\sum_{i} \frac{\partial x^{i}}{\partial y^{s}} \frac{\partial}{\partial a^{i}{ }_{j}} \\
& d y^{i}=\sum_{j} \frac{\partial y^{i}}{\partial x^{j}} d x^{j}, \quad d b^{i}{ }_{j}=\sum_{k, l} \frac{\partial^{2} y^{i}}{\partial x^{k} \partial x^{l}} a_{j}^{l} d x^{k}+\sum_{l} \frac{\partial y^{i}}{\partial x^{l}} d a^{l}{ }_{j} .
\end{aligned}
$$

From (2.5), (2.8), (2.11), (2.13) and (2.14), we obtain

$$
\begin{aligned}
X^{\star} & =\sum_{i} \xi^{i}\left(\partial / \partial x^{i}\right)_{u}-\sum_{i, l} \xi^{i} a_{j}^{l}(u) \Gamma_{i l}^{k}(p)\left(\partial / \partial a^{k}{ }_{j}\right)_{u} \\
& =\sum_{i} \eta^{i}\left(\partial / \partial y^{i}\right)_{u}-\sum_{i, l} \eta^{i} b_{j}^{l}(u) \tilde{\Gamma}_{i l}^{k}(p)\left(\partial / \partial b^{k}{ }_{j}\right)_{u} .
\end{aligned}
$$

This shows that the horizontal subspace does not depend on the choice of the local coordinate system around the point $u \in L(M)$, which is rarely seen. Thus we have

Theorem 2.2. Let $u \in L(M)$ be an arbitrarily given point, $\left(x^{1}, \ldots, x^{n}\right)$ a coordinate system on a neighborhood $U \subset M$ which contains $\pi(u)=p$, and $\left(x^{1}, \ldots, x^{n}, a^{1} 1, \ldots, a_{n}^{n}\right)$ the coordinate system on $\pi^{-1}(U) \subset L(M)$. Let $\Gamma_{j k}^{i}$ be the Christoffel's symbols on $U$ which are defined by a linear connection $\nabla: \mathfrak{X}(M) \times \mathfrak{X}(M) \rightarrow \mathfrak{X}(M)$. Then the horizontal subspace $Q_{u}$ of $T_{u} L(M)$ at the point $u$ is given as follows: 
$Q_{u}=\left\{\sum_{i} \xi^{i}\left(\partial / \partial x^{i}\right)_{u}-\sum_{i, l} \xi^{i} a_{j}^{l}(u) \Gamma_{i l}^{k}(p)\left(\frac{\partial}{\partial a^{k}{ }_{j}}\right)_{u} \mid\right.$ each $\left.\xi^{i} \in \mathbb{R}\right\}$

\section{References}

[1] S. Helgason, Differential Geometry, Lie Groups and Symmetric Spaces, Academic Press, New York, 1978.

[2] S. Kobayashi and K. Nomizu, Foundation of Differential Geometry, Vol.I, Wiley-Interscience, New York, 1963.

[3] I. Mogi and M. Itoh, Differential Geometry and Gauge Theory (in Japanese), Kyoritsu Publ., 1986.

[4] Walter A. Poor, Differential Geometric Structures, McGraw-Hill, Inc., 1081.

\section{Joon-Sik Park}

Department of Mathematics, Busan University of Foreign Studies, Pusan 608-738, Korea.

E-mail: iohpark@bufs.ac.kr 